\title{
Analysis of a time-delayed free boundary problem for solid tumor growth with angiogenesis and direct influence of inhibitors
}

\section{Shihe $X u^{1 *}$ and Dan Su}

"Correspondence:

shihe56789@163.com

${ }^{1}$ School of Mathematics and

Statistics, Zhaoging University,

Zhaoging, P.R. China

Full list of author information is

available at the end of the article

\section{Springer}

\begin{abstract}
In this paper we consider a free boundary problem for tumor growth under direct effect of inhibitors with angiogenesis and time delays in proliferation. The existence and uniqueness of the steady state solution is studied. The asymptotic behavior of steady state solution is proved, and the condition under which the tumor will tend to disappear is given. Finally, we also discuss the effects of the concentration of external inhibitors, the concentration of external nutrients, and the consumption rate of nutrients and inhibitors on the growth of tumors. The results show that under certain conditions the tumor will eventually disappear or will tend to a steady state. The increase of inhibitor concentration (or consumption rate) will lead to the reduction of the radius of the tumor, and the increase of nutrient concentration (or consumption rate) will lead to the increase of the radius of the tumor.
\end{abstract}

MSC: 35K57; 35Q92; 35R35

Keywords: Tumor growth; Angiogenesis; Time delay; Free boundary problem; Stability

\section{Introduction}

In the last forty years, many papers devoted to studying mathematical models to describe the process of tumor growth have appeared (see, e.g., [1, 2, 4-6, 12, 14-19]). The process of tumor growth has several different stages, generally speaking, from the early stage without angiogenesis or (and) necrotic core inside (see, e.g., $[6,8-10,13,20]$ ) to the process of necrotic core or (and) angiogenesis formation (see, e.g., [4, 7, 12, 19, 23]). Experiments suggest that changes in the proliferation rate can trigger changes in apoptotic cell loss. But these changes do not occur instantaneously: they are mediated by growth factors expressed by the tumor cells (see [5]). Following this idea, the study of mathematical models with time delays for tumor growth has drawn some attention (see, e.g., $[10,11,21]$ and the references cited therein). In this paper we consider a free boundary problem for tumor growth under direct effect of inhibitors with angiogenesis and time delays in the proliferation process.

C) The Author(s) 2020. This article is licensed under a Creative Commons Attribution 4.0 International License, which permits use, sharing, adaptation, distribution and reproduction in any medium or format, as long as you give appropriate credit to the original author(s) and the source, provide a link to the Creative Commons licence, and indicate if changes were made. The images or other third party material in this article are included in the article's Creative Commons licence, unless indicated otherwise in a credit line to the material. If material is not included in the article's Creative Commons licence and your intended use is not permitted by statutory regulation or exceeds the permitted use, you will need to obtain permission directly from the copyright holder. To view a copy of this licence, visit http://creativecommons.org/licenses/by/4.0/. 
Firstly, we introduce the mathematical model which has three unknown functions:

- $\sigma(r, t)$ - the nutrient concentration at radius $r$ and time $t$,

- $\beta(r, t)$-the inhibitor concentration at radius $r$ and time $t$,

- $R(t)$-the outer tumor radius at time $t$.

Nutrient concentration $\sigma$ and inhibitor concentration $\beta$ satisfy the following two reaction-diffusion equations respectively:

$$
\begin{array}{ll}
c_{1} \frac{\partial \sigma}{\partial t}=\frac{1}{r^{2}} \frac{\partial}{\partial r}\left(r^{2} \frac{\partial \sigma}{\partial r}\right)-\lambda \sigma, & 0<r<R(t), t>0, \\
c_{2} \frac{\partial \beta}{\partial t}=\frac{1}{r^{2}} \frac{\partial}{\partial r}\left(r^{2} \frac{\partial \beta}{\partial r}\right)-\gamma \beta, & 0<r<R(t), t>0,
\end{array}
$$

where $c_{i}=T_{\text {diffusion }}^{i} / T_{\text {growth }}(i=1,2)$ are positive constants which represent the ratio of the nutrient/inhibitors diffusion time scale to the tumor growth (e.g., tumor doubling) time scale (for details, please see $[6,11,13]), T_{\text {diffusion }}^{1}$ represents the ratio of the nutrient diffusion time scale, and $T_{\text {diffusion }}^{2}$ represents the ratio of the inhibitors diffusion time scale. $\lambda$ is the consumption rate of nutrient and $\gamma$ is the consumption rate of inhibitors.

By the mass conservation law, the rate of tumor volume change is equal to the rate of tumor volume growth caused by tumor cell proliferation minus the rate of tumor volume reduction caused by tumor cell natural apoptosis and tumor cell apoptosis caused by inhibitors. Thus, the changes of $R$ satisfy

$$
\frac{d}{d t}\left(\frac{4 \pi R^{3}}{3}\right)=S-Q-I
$$

where $S$ and $Q$ denote the rates of proliferation and natural apoptosis respectively and $I$ represents the apoptosis rate caused by inhibitors.

Assume that the proliferation rate is proportional to the local nutrient concentration. The coefficient of proportionality is denoted by $\mu$, then

$$
S=4 \pi \int_{0}^{R(t-\tau)} \mu \sigma(r, t-\tau) r^{2} d r
$$

where $\tau$ ( $\approx 24$ hours) is the time delay in cell proliferation process. We assume that the natural apoptosis rate is a constant denoted by $a$. For the convenience of mathematical discussion, let $a=\mu \tilde{\sigma}$, so the apoptosis rate and proliferation rate of cells have the common factor $\mu$, and

$$
Q=4 \pi \int_{0}^{R(t)} \mu \tilde{\sigma} r^{2} d r
$$

Assume the apoptotic cell loss rate caused by inhibitors is proportional to the local inhibitors concentration. The coefficient of proportionality is denoted by $v$, thus

$$
I=4 \pi \int_{0}^{R(t)} \nu \beta(r, t) r^{2} d r .
$$


Since nutrients enter the tumors by their vascular system, following the ideas of $[12,19$, 23], we will consider the problem with the following boundary conditions:

$$
\begin{array}{ll}
\frac{\partial \sigma}{\partial r}+\alpha_{1}(\sigma-\bar{\sigma})=0, & r=R(t), \\
\frac{\partial \beta}{\partial r}+\alpha_{2}(\beta-\bar{\beta})=0, & r=R(t),
\end{array}
$$

where $\alpha_{i}(i=1,2)$ are positive constants which depend on the density of the blood vessels; $\bar{\sigma}$ denotes the external concentration of nutrients, and $\bar{\beta}$ denotes the external concentration of inhibitors. In [12], the authors consider a more general boundary condition where $\alpha$ is assumed to be a positive function of $t$, but they do not consider the effects of inhibitors and the time delay. In [19], a mathematical model with effect of inhibitor is studied where the natural apoptosis rate is assumed to be 0 , and there is no discussion of the effect of the inhibitor on tumor growth. In [23], the authors consider a problem without the effect of inhibitors and the time delay, but with surface tension. The authors prove that the free boundary problem has a unique radial stationary solution which is asymptotically stable for large surface tension coefficients, whereas unstable for small surface tension coefficients.

Consider the problem together with the following initial value function:

$$
R(t)=\varphi(t), \quad-\tau \leq t \leq 0 .
$$

From [6,9] we know that $T_{\text {diffusion }}^{i} \approx 1 \min (i=1,2)$ and $T_{\text {growth }} \approx 1$ day, so that $c_{i} \ll 1$ $(i=1,2)$. For this reason and simplicity, we only consider the limiting case where $c_{i}=0$ $(i=1,2)$ in this paper. Since the tumor has only one vascular system, it is reasonable to assume that $\alpha_{1}=\alpha_{2}:=\alpha$. Thus, in this paper we study a delayed mathematical model for tumor growth with angiogenesis as follows:

$$
\begin{aligned}
& \Delta_{r} \sigma-\lambda \sigma=0, \quad 0<r<R(t), t>0, \\
& \frac{\partial \sigma}{\partial r}+\alpha(\sigma-\bar{\sigma})=0, \quad r=R(t), t>0, \\
& \Delta_{r} \beta-\gamma \beta=0, \quad 0<r<R(t), t>0, \\
& \frac{\partial \beta}{\partial r}+\alpha(\beta-\bar{\beta})=0, \quad r=R(t), t>0, \\
& \frac{d}{d t}\left(\frac{4 \pi R^{3}(t)}{3}\right) \\
& \quad=4 \pi\left(\int_{0}^{R_{\tau}} \mu \sigma_{\tau} r^{2} d r-\int_{0}^{R(t)} \nu \beta(r, t) r^{2} d r-\int_{0}^{R(t)} \mu \tilde{\sigma} r^{2} d r\right), \quad t>0, \\
& R(t)=\varphi(t), \quad-\tau \leq t \leq 0,
\end{aligned}
$$

where $\sigma_{\tau}=\sigma(r, t-\tau), R_{\tau}=R(t-\tau)$, and

$$
\Delta_{r} \cdot=\frac{1}{r^{2}} \frac{\partial}{\partial r}\left(r^{2} \frac{\partial \cdot}{\partial r}\right)
$$


The paper is arranged as follows. In Sect. 2, we prove the existence and uniqueness of the solution to Eqs. (1.10)-(1.15). Section 3 is devoted to the steady state solutions and their stability. In Sect. 4, we discuss the effects of inhibitor and nutrient parameters on tumor growth.

\section{Existence and uniqueness of the nonnegative solution to problem}

\section{(1.10)-(1.15)}

We introduce the following functions which will be used in our following analysis:

$$
p(x)=\frac{x \operatorname{coth} x-1}{x^{2}}, \quad g(x)=x p(x)=\operatorname{coth} x-\frac{1}{x}, \quad h(x)=x^{3} p(x)
$$

and

$$
D(x)=\frac{h(x)}{\alpha+g(x)}, \quad L(x)=\frac{l(\vartheta x)}{l(x)}, \quad l(x)=\frac{\alpha p(x)}{\alpha+g(x)} .
$$

\section{Lemma 2.1}

(1) $p^{\prime}(x)<0$ for all $x>0$, and $\lim _{x \rightarrow 0+} p(x)=\frac{1}{3}, \lim _{x \rightarrow \infty} p(x)=0$.

(2) $h(x)$ and $g(x)$ are strictly monotone increasing for $x>0$, and

$$
g(0)=0, \quad \lim _{x \rightarrow \infty} g(x)=1, \quad g^{\prime}(0)=1 / 3
$$

(3) For any $\alpha>0, D(x)$ is strictly monotone increasing for $x>0$.

(4) When $0<\vartheta<1, L(x)$ is strictly monotone increasing for $x>0$; when $\vartheta>1, L(x)$ is strictly monotone decreasing for $x>0$.

Proof (1) Please see the last eight lines in the proof of Theorem 2.1 on page 266 in [13].

(2) The proof of monotonicity of $h$ can be found on page 533, lines 5-10 in [10]. For the proof of the properties and monotonicity of $g$, please see Lemma 2.1 in [12].

(3) Direct computation yields

$$
\begin{aligned}
D^{\prime}(x) & =\frac{h^{\prime}(x)(\alpha+g(x))-g^{\prime}(x) h(x)}{(\alpha+g(x))^{2}} \\
& =\frac{\alpha h^{\prime}(x)}{(\alpha+g(x))^{2}}+\left(\frac{h(x)}{g(x)}\right)^{\prime} \frac{g^{2}(x)}{(\alpha+g(x))^{2}} \\
& =\frac{\alpha h^{\prime}(x)}{(\alpha+g(x))^{2}}+2 x \frac{g^{2}(x)}{(\alpha+g(x))^{2}}>0,
\end{aligned}
$$

where we have used the facts that $h^{\prime}(x)>0$ and $g(x)>0$. Thus, $D(x)$ is strictly monotone increasing for $x>0$.

(4) First, we prove $\frac{x l^{\prime}(x)}{l(x)}$ is strictly monotone decreasing in $x$. Since

$$
\frac{x l^{\prime}(x)}{l(x)}=\frac{x\left(\alpha p^{\prime}(x)-p^{2}(x)\right)}{p(x)(\alpha+x p(x))}
$$

by a direct computation, we have

$$
\left(\frac{x l^{\prime}(x)}{l(x)}\right)^{\prime}=\frac{J(x)}{p^{2}(\alpha+x p)^{2}}
$$


where

$$
\left.J(x)=\alpha^{2}\left[\left(p^{\prime}+x p^{\prime \prime}\right) p-x\left(p^{\prime}\right)^{2}\right)\right]+\alpha\left[p\left(x^{2} p^{\prime \prime} p-2 x p^{\prime} p-2 x^{2}\left(p^{\prime}\right)^{2}-p^{2}\right)+x p^{2} p^{\prime}\right] .
$$

Noticing that

$$
\left(p^{\prime}+x p^{\prime \prime}\right) p-x\left(p^{\prime}\right)^{2}=p^{2}\left(\frac{x p^{\prime}}{p}\right)^{\prime}<0
$$

and

$$
p\left(x^{2} p^{\prime \prime} p-2 x p^{\prime} p-2 x^{2}\left(p^{\prime}\right)^{2}-p^{2}\right)+x p^{2} p^{\prime}=x p^{3}\left(\frac{x p^{\prime}}{p}\right)^{\prime}-2 p\left[(x p)^{\prime}\right]^{2}<0
$$

where we have used the facts that $\left(\frac{x p^{\prime}}{p}\right)^{\prime}<0$ (see Lemma $\left.2.2[21]\right), p>0$, and $(x p)^{\prime}>0$ (see Lemma 2.1(2)) for $x>0$, we can get $J(x)<0$ for $\alpha>0, x>0$. Therefore $\left(\frac{x l^{\prime}(x)}{l(x)}\right)^{\prime}<0$ for $x>0$, which implies that $\frac{x l^{\prime}(x)}{l(x)}$ is strictly monotone decreasing in $x$.

Next, by a direct computation, one can get

$$
\begin{aligned}
L^{\prime}(x) & =\left(\frac{l(\vartheta x)}{l(x)}\right)^{\prime}=\frac{\vartheta l^{\prime}(\vartheta x) l(x)-l^{\prime}(x) l(\vartheta x)}{l^{2}(x)} \\
& =\frac{l(\vartheta x)}{x l(x)}\left(\frac{\vartheta x l^{\prime}(\vartheta x)}{l(\vartheta x)}-\frac{x l^{\prime}(x)}{l(x)}\right) .
\end{aligned}
$$

Then, it follows that

$$
L^{\prime}(x) \begin{cases}>0, & 0<\vartheta<1, \\ =0, & \vartheta=1, \\ <0, & \vartheta>1 .\end{cases}
$$

This completes the proof.

Theorem 2.2 For any nonnegative initial value function $\varphi(t), t \in[-\tau, 0]$, there exists a unique nonnegative solution to problem (1.10)-(1.15).

Proof The solution to (1.10)-(1.13) is

$$
\sigma(r, t)=\frac{\alpha \bar{\sigma}}{\alpha+g(\sqrt{\lambda} R)} \frac{f(\sqrt{\lambda} r)}{f(\sqrt{\lambda} R(t))}, \quad \beta(r, t)=\frac{\alpha \bar{\beta}}{\alpha+g(\sqrt{\gamma} R)} \frac{f(\sqrt{\gamma} r)}{f(\sqrt{\gamma} R(t))},
$$

where $f(x)=\sinh x / x$. Substituting (2.2) into (1.14) and setting $x=\sqrt{\lambda} R$, one can get

$$
\frac{d x}{d t}=\mu \bar{\sigma} x\left[\frac{\alpha}{\alpha+g\left(x_{\tau}\right)} \frac{\left.x_{\tau}^{3} p\left(x_{\tau}\right)\right)}{x^{3}}-\frac{\nu \bar{\beta}}{\mu \bar{\sigma}} \frac{\alpha p(\vartheta x)}{\alpha+g(\vartheta x)}-\frac{\tilde{\sigma}}{3 \bar{\sigma}}\right],
$$

where $\vartheta=\sqrt{\gamma / \lambda}, x_{\tau}=x(t-\tau)$ and $p, g$ are as before. Let $y=x^{3}$. Then Eq. (2.3) takes the following form:

$$
\frac{d y}{d t}=3 \mu \bar{\sigma} y(t-\tau) l(\sqrt[3]{y(t-\tau)})-[3 \nu \bar{\beta} l(\vartheta \sqrt[3]{y(t)})+\tilde{\sigma}] y(t)=:-F(y(t)+H(y(t-\tau))),
$$


where

$$
F(y)=[3 v \bar{\beta} l(\vartheta \sqrt[3]{y(t)})+\tilde{\sigma}] y(t)=\left[\frac{3 v \alpha \bar{\beta}}{\vartheta^{3}} D(\vartheta \sqrt[3]{y(t)})+\tilde{\sigma}\right] y(t)
$$

and

$$
H(y(t-\tau))=3 \mu \bar{\sigma} y(t-\tau) l(\sqrt[3]{y(t-\tau)})=3 \mu \alpha \bar{\sigma} D(\sqrt[3]{y(t-\tau)}) .
$$

Accordingly, the initial condition is as follows:

$$
y_{0}(t)=\sqrt{\lambda^{3}} \varphi^{3}(t), \quad-\tau \leq t \leq 0 .
$$

Thus we only need to prove the existence and uniqueness of the solution to (2.4) and (2.5).

By Lemma 2.1(3), we easily get

$$
F^{\prime}(y)=\frac{v \alpha \bar{\beta}}{\vartheta^{2}} D^{\prime}(\vartheta \sqrt[3]{y(t)}) \sqrt[3]{y(t)}+\left[\frac{3 v \alpha \bar{\beta}}{\vartheta^{3}} D(\vartheta \sqrt[3]{y(t)})+\tilde{\sigma}\right]>0
$$

for $y>0$. If $y_{0}(t)$ is continuous for $t \in[-\tau, 0]$, by Lemma 2.3 in [22], one can get that there exists a unique solution to Eq. (2.4) on $R^{+}$. From Lemma 2.1(3) we know that $H(y) \geq 0$ for all $y \geq 0$, by Theorem 1.1 in [3], it is easy to get the solution of problem (2.4), (2.5) is nonnegative on $R^{+}$. This completes the proof.

\section{The steady state solutions and their stability}

Lemma 3.1 Assume that $\mu \bar{\sigma}>\nu \bar{\beta}+\mu \tilde{\sigma}$. The problem has at least one unique positive steady state solution $x=x_{s}$ for any $\alpha>0$. Assume further that $0<\vartheta<1$, the problem has a unique positive steady state solution $x=x_{s}$ for each $\alpha>0$. Moreover, $x_{s}$ is related to $\alpha$ and $\eta$. If we take $x_{s}$ as a function of $\alpha$ and $\eta$, we write it as $x_{s}=x_{s}(\alpha, \eta)$. Then $x_{s}(\alpha, \eta)$ is strictly monotone increasing in $\alpha$ and strictly monotone decreasing in $\eta$, where $\eta=\tilde{\sigma} / \bar{\sigma}$. Furthermore, for each $\eta \in(0,1), x_{s} \rightarrow x_{s}^{D}$, as $\alpha \rightarrow \infty$, where $x_{s}^{D}$ is the unique positive solution to

$$
p(x)-\frac{\nu \bar{\beta}}{\mu \bar{\sigma}} p(\vartheta x)=\eta / 3 .
$$

Proof The steady state solutions satisfy the following equation:

$$
\frac{\alpha}{\alpha+g(x)} p(x)-\frac{\nu \bar{\beta}}{\mu \bar{\sigma}} \frac{\alpha}{\alpha+g(\vartheta x)} p(\vartheta x)=\frac{\eta}{3}
$$

where $\eta=\tilde{\sigma} / \bar{\sigma}$. Rewrite (3.2) in the following form:

$$
\Theta(x):=l(x) \Lambda(x)=l(x)\left(1-\frac{\nu \bar{\beta}}{\mu \bar{\sigma}} L(x)-\frac{\eta}{3 l(x)}\right)=0,
$$

where $\Lambda(x)=1-\frac{\nu \bar{\beta}}{\mu \bar{\sigma}} L(x)-\frac{\eta}{3 l(x)}, L(x)$ and $l(x)$ are as before. Since

$$
\lim _{x \rightarrow 0} \Lambda(x)=1-\frac{\nu \bar{\beta}}{\mu \bar{\sigma}}-\eta, \quad \lim _{x \rightarrow \infty} \Lambda(x)=-\infty
$$


by the mean value theorem, one can get that there exists at least one positive solution $x=x_{s}$ to $(3.2)$ if

$$
1-\frac{\nu \bar{\beta}}{\mu \bar{\sigma}}-\eta>0 \quad \Leftrightarrow \quad \mu \bar{\sigma}>\nu \bar{\beta}+\mu \tilde{\sigma} .
$$

Since

$$
l^{\prime}(x)=\frac{\alpha\left[\alpha p^{\prime}-p^{2}\right]}{(\alpha+x p)^{2}}<0,
$$

then, if $0<\vartheta<1$, by Lemma 2.1(4), we have

$$
\Lambda^{\prime}(x)=-\frac{\nu \bar{\beta}}{\mu \bar{\sigma}} L^{\prime}(x)+\frac{\eta l^{\prime}(x)}{(3 l(x))^{2}}<0 .
$$

Therefore, there exists a unique positive solution $x=x_{s}$ to (3.2) if condition (3.4) holds.

Next we prove that Eq. (3.1) has a unique solution $x_{s}^{D}$. Since $p(x)>0$, it suffices to prove that

$$
1-\frac{\nu \bar{\beta}}{\mu \bar{\sigma}} \frac{p(\vartheta x)}{p(x)}-\frac{\eta}{3 p(x)}=0
$$

has a unique solution $x_{s}^{D}$. By a simple computation, for $0<\vartheta<1$, one can get

$$
\left(\frac{p(\vartheta x)}{p(x)}\right)^{\prime}=\frac{p(\vartheta x)}{x p(x)}\left(\frac{\vartheta x p^{\prime}(\vartheta x)}{p(\vartheta x)}-\frac{x p^{\prime}(x)}{p(x)}\right)>0,
$$

since $\left(\frac{x p^{\prime}}{p}\right)^{\prime}<0$ (see Lemma $2.2[21]$ ). Therefore, noting the facts $p(x)>0$ and $p^{\prime}(x)<0$ for $x>0$, the function $1-\frac{v \bar{\beta}}{\mu \bar{\sigma}} \frac{p(\vartheta x)}{p(x)}-\frac{\eta}{3 p(x)}$ on the left-hand side of (3.6) is monotone decreasing in $x$. Then Eq. (3.6) has a unique solution $x_{s}^{D}$ follows from the facts that

$$
\lim _{x \rightarrow 0}\left(1-\frac{\nu \bar{\beta}}{\mu \bar{\sigma}} \frac{p(\vartheta x)}{p(x)}-\frac{\eta}{3 p(x)}\right)=1-\frac{\nu \bar{\beta}}{\mu \bar{\sigma}}-\eta>0
$$

and

$$
\lim _{x \rightarrow \infty}\left(1-\frac{\nu \bar{\beta}}{\mu \bar{\sigma}} \frac{p(\vartheta x)}{p(x)}-\frac{\eta}{3 p(x)}\right)=-\infty<0 .
$$

If we take $x_{s}$ as a function of $\alpha$ and $\eta$, we write it as $x_{s}=x_{s}(\alpha, \eta)$. Using the derivative rule of implicit function, by taking the derivative of $\alpha$ on both sides of the equation $\Lambda\left(x_{s}(\alpha, \eta)\right)=$ 0 , one can get

$$
-\frac{\nu \bar{\beta}}{\mu \bar{\sigma}} L_{x_{s}}^{\prime} x_{\alpha}^{\prime}-\frac{\nu \bar{\beta}}{\mu \bar{\sigma}} L_{\alpha}^{\prime}+\frac{\eta l_{x_{s}}^{\prime}}{3 l^{2}}\left(l_{x_{s}}^{\prime} x_{\alpha}^{\prime}+l_{\alpha}^{\prime}\right)=0
$$

where $x_{\alpha}^{\prime}:=\frac{\partial x_{s}}{\partial \alpha}(\alpha, \eta)$, and

$$
l_{\alpha}^{\prime}=\frac{\partial l}{\partial \alpha}\left(x_{s}, \alpha, \eta\right)=\frac{g}{(\alpha+g)^{2}}>0,
$$




$$
\begin{aligned}
& L_{\alpha}^{\prime}=\frac{\partial L}{\partial \alpha}\left(x_{s}, \alpha, \eta\right)=\frac{x p^{2}\left(x p^{\prime}-p\right)}{p^{2}(\alpha+x p)}<0, \\
& l_{x_{s}}^{\prime}=\frac{\partial l}{\partial x_{s}}\left(x_{s}, \alpha, \eta\right)=\frac{-g^{\prime}}{(a+g)^{2}}<0 .
\end{aligned}
$$

From Lemma 2.1(4), we know that $L_{x_{\mathrm{s}}}^{\prime}>0$. It follows that

$$
\frac{\partial x_{s}}{\partial \alpha}=\frac{-\frac{\nu \bar{\beta}}{\mu \bar{\sigma}} L_{\alpha}^{\prime}+\frac{\eta l_{\alpha}^{\prime}}{3 l^{2}}}{\frac{\nu \bar{\beta}}{\mu \bar{\sigma}} L_{x_{s}}^{\prime}-\frac{\eta l_{x_{s}}^{\prime}}{3 l^{2}}}>0 .
$$

Similarly, by the derivative rule of implicit function, we take the derivative of $\eta$ on both sides of the equation $\Lambda\left(x_{s}(\alpha, \eta)\right)=0$ and find the derivative of $\eta$, which is given by

$$
\frac{\partial x_{s}}{\partial \eta}=\frac{l}{-3 A l^{2} L_{x_{s}}^{\prime}+\eta l_{x_{s}}^{\prime}}<0
$$

where $A=\frac{\nu \bar{\beta}}{\mu \bar{\sigma}}$ and $l_{x_{s}}^{\prime}$ is as before, where we used the facts that $L_{x_{s}}^{\prime}>0$ and $l_{x_{s}}^{\prime}<0$ for $\vartheta \in(0,1)$. Therefore $x_{s}(\alpha, \eta)$ is strictly monotone increasing in $\alpha$ and strictly monotone decreasing in $\eta$, where $\eta=\tilde{\sigma} / \bar{\sigma}$. Then it follows that, for each $\eta \in(0,1), x_{s} \rightarrow x_{s}^{D}$ as $\alpha \rightarrow \infty$, where $x_{s}^{D}$ is the unique positive solution to

$$
p(x)-\frac{\nu \bar{\beta}}{\mu \bar{\sigma}} p(\vartheta x)=\eta / 3
$$

This completes the proof.

Lemma 3.2 ([10]) Consider the initial value problem of a delay differential equation

$$
\begin{aligned}
& \dot{x}(t)=G(x(t), x(t-\tau)), \quad t>0, \\
& x(t)=x^{0}(t), \quad-\tau \leq t \leq 0 .
\end{aligned}
$$

Assuming that the function $G$ is defined and continuously differentiable in $R_{+} \times R_{+}$and strictly monotone increasing in the second variable, we have following results:

(1) If $x_{s}$ is a positive solution of the equation $G(x, x)=0$ such that $G(x, x)>0$ for $x$ less than but near $x_{s}, G(x, x)<0$ for $x$ greater than but near $x_{s}$. Let $(c, d)$ be the (maximal) interval containing only the root $x_{s}$ of the equation $G(x, x)=0$. If $x(t)$ is the solution of the problem of (3.8), (3.9) and $x^{0}(t) \in C[-\tau, 0], c<x^{0}(t)<d$ for $-\tau \leq t \leq 0$, then

$$
\lim _{t \rightarrow \infty} x(t)=x_{s}
$$

(2) If $G(x, x)<0$ for all $x>0$, then

$$
\lim _{t \rightarrow \infty} x(t)=0 .
$$

Theorem 3.3 Assume $\vartheta \in(0,1)$. For any nonnegative initial value $\varphi(t),-\tau \leq t \leq 0$,

(1) if $\mu \bar{\sigma}>\nu \bar{\beta}+\mu \tilde{\sigma}$, the solution of (2.3) converges to $x_{s}$ as $t \rightarrow \infty$;

(2) if $\mu \bar{\sigma}<\nu \bar{\beta}+\mu \tilde{\sigma}$, the solution of (2.3) converges to 0 as $t \rightarrow \infty$. 
Proof Let

$$
G(x, y)=\frac{\alpha}{\alpha+g(y)} \frac{\left.y^{3} p(y)\right)}{x^{3}}-\frac{\nu \bar{\beta}}{\mu \bar{\sigma}} \frac{\alpha p(\vartheta x)}{\alpha+g(\vartheta x)}-\eta / 3
$$

where $\eta=\tilde{\sigma} / \bar{\sigma}$. Then

$$
G(x, x)=\Theta(x)=l(x) \Lambda(x)=l(x)\left(1-\frac{\nu \bar{\beta}}{\mu \bar{\sigma}} L(x)-\frac{\eta}{3 l(x)}\right) .
$$

Direct computation yields

$$
\frac{\partial G}{\partial y}=\frac{\alpha}{x^{3}} \frac{\partial F}{\partial y}>0
$$

thus $G$ is strictly monotone increasing in the second variable $y$.

By Lemma 2.1(4) and (3.5), we know that $\Lambda(x)$ is strictly monotone decreasing, and by Lemma 2.1(1) (2), one can easily get

$$
\lim _{x \rightarrow 0} \Lambda(x)=1-\frac{\nu \bar{\beta}}{\mu \bar{\sigma}}-\eta, \quad \lim _{x \rightarrow \infty} \Lambda(x)=-\infty .
$$

If $\mu \bar{\sigma}>\nu \bar{\beta}+\mu \tilde{\sigma}$, we can get that there exists a unique positive constant $x_{s}$ such that $x_{s}$ is the unique positive solution of the equation $G(x, x)=0$. Moreover, $G(x, x)>0$ for $x<x_{s}$; $G(x, x)<0$ for $x>x_{s}$. By Lemma 3.2(1), we get that

$$
\lim _{t \rightarrow \infty} R(t)=x_{s}
$$

holds for any nonnegative initial value $\varphi(t),-\tau \leq t \leq 0$.

(2) By the facts that $\Lambda(x)$ is strictly monotone decreasing and

$$
\lim _{x \rightarrow 0} \Lambda(x)=1-\frac{\nu \bar{\beta}}{\mu \bar{\sigma}}-\eta, \quad \lim _{x \rightarrow \infty} \Lambda(x)=-\infty,
$$

if $\mu \bar{\sigma}<\nu \bar{\beta}+\mu \tilde{\sigma}$, one can get that

$$
G(x, x)=l(x) \Lambda(x)<0
$$

since $\Lambda(x)<0$. By Lemma 3.2(2), we get that

$$
\lim _{t \rightarrow \infty} R(t)=0
$$

holds for any nonnegative initial value $\varphi(t),-\tau \leq t \leq 0$. This competes the proof.

\section{Effects of inhibitor and nutrient parameters on tumor growth}

We consider the relevant parameters of inhibitors and nutrients as independent variables and write (2.3) as follows:

$$
\frac{d x}{d t}=\Phi\left(x, x_{\tau}, \bar{\sigma}, \bar{\beta}, \vartheta\right)
$$


where

$$
\Phi(x, y, \bar{\sigma}, \bar{\beta}, \vartheta)=\mu \bar{\sigma} x\left[\frac{\alpha}{\alpha+g(y)} \frac{\left.y^{3} p(y)\right)}{x^{3}}-\frac{\nu \bar{\beta}}{\mu \bar{\sigma}} \frac{\alpha p(\vartheta x)}{\alpha+g(\vartheta x)}-\frac{\tilde{\sigma}}{3 \bar{\sigma}}\right] .
$$

By a simple computation, we have

$$
\begin{aligned}
& \frac{\partial \Phi}{\partial \bar{\alpha}}=\frac{v \bar{\beta}}{\bar{\sigma}} \frac{\alpha x p(\vartheta x)}{\alpha+g(\vartheta x)}+\frac{\mu \tilde{\sigma}}{3 \bar{\sigma}} x>0, \\
& \frac{\partial \Phi}{\partial \bar{\beta}}=-v \frac{\alpha x p(\vartheta x)}{\alpha+g(\vartheta x)}<0
\end{aligned}
$$

and

$$
\frac{\partial \Phi}{\partial \vartheta}=-v \bar{\beta} \alpha x \frac{\vartheta\left[\alpha p^{\prime}(\vartheta x)-p^{2}(\vartheta x)\right]}{[\alpha+g(\vartheta x)]^{2}}>0
$$

Assume $\bar{\sigma}_{1}>\bar{\sigma}_{2}$ and the other parameters $\bar{\beta}, \mu, v, \vartheta, \tilde{\sigma}$, and $\alpha$ being fixed. Under the conditions of the above parameters, where $\bar{\sigma}_{1}>\bar{\sigma}_{2}$ and $\bar{\beta}_{1}=\bar{\beta}_{2}, \vartheta_{1}=\vartheta_{2}, \mu_{1}=\mu_{2}, v_{1}=v_{2}, \tilde{\sigma}_{1}=\tilde{\sigma}_{2}$, and $\alpha_{1}=\alpha_{2}$, let $x_{1}(t)$ and $x_{2}(t)$ be the solutions to problem (4.1) with the same initial value function $\varphi$ respectively. Since

$$
\frac{\partial \Phi}{\partial y}=\frac{\mu \bar{\sigma} \alpha}{x^{2}} \frac{\partial F}{\partial y}>0
$$

by the comparison principle (see, e.g., Lemma 3.1 [10]), one can get that $x_{1}(t) \geq x_{2}(t)$ for all $t>0$. Biologically speaking, if other conditions remain unchanged, increasing the concentration of external nutrients will increase the radius of tumors.

Similarly, by the comparison principle, (4.2), and (4.3), we can get:

- If other conditions remain unchanged, increasing the concentration of external inhibitors will reduce the radius of tumors.

- If other conditions remain unchanged, increasing $\vartheta$ will increase the radius of tumors.

\section{Acknowledgements}

The authors would like to thank the editor and the two referees for their very helpful suggestions on modification of the original manuscript.

Funding

The work of the first author is partially supported by NNSF of China (11301474), NSF of Guangdong Province (2018A030313536).

Availability of data and materials

Not applicable.

Competing interests

The authors declare that they have no competing interests.

Authors' contributions

The two authors completed the paper together. Both authors read and approved the final manuscript.

\section{Author details}

${ }^{1}$ School of Mathematics and Statistics, Zhaoging University, Zhaoging, P.R. China. ${ }^{2}$ Foundation Education Institute,

Lingnan Normal University, Zhanjiang, P.R. China. 


\section{Publisher's Note}

Springer Nature remains neutral with regard to jurisdictional claims in published maps and institutional affiliations.

Received: 12 June 2019 Accepted: 27 February 2020 Published online: 05 March 2020

\section{References}

1. Bianca, C., Pappalardo, F., Motta, S., Ragusa, M.A.: Persistence analysis in a Kolmogorov-type model for cancer-immune system competition. AIP Conf. Proc. 1558, 1797-1800 (2013)

2. Bianca, C., Pennisi, M., Motta, S., Ragusa, M.A.: Immune system network and cancer vaccine. AIP Conf. Proc. 1389, 945-948 (2011)

3. Bodnar, M.: The nonnegativity of solutions of delay differential equations. Appl. Math. Lett. 13, 91-95 (2000)

4. Bodnar, M., Foryś, U.: Time delay in necrotic core formation. Math. Biosci. Eng. 2, 461-472 (2005)

5. Byrne, H.: The effect of time delays on the dynamics of avascular tumor growth. Math. Biosci. 144, 83-117 (1997)

6. Byrne, H., Chaplain, M.: Growth of nonnecrotic tumors in the presence and absence of inhibitors. Math. Biosci. 130, 151-181 (1995)

7. Cui, S.: Analysis of a mathematical model for the growth of tumors under the action of external inhibitors. J. Math. Biol. 44, 395-426 (2002)

8. Cui, S.: Analysis of a free boundary problem modeling tumor growth. Acta Math. Sin. 21, 1071-1082 (2005)

9. Cui, S., Friedman, A.: Analysis of a mathematical model of the effect of inhibitors on the growth of tumors. Math. Biosci. 164, 103-137 (2000)

10. Cui, S., Xu, S.: Analysis of mathematical models for the growth of tumors with time delays in cell proliferation. J. Math. Anal. Appl. 336, 523-541 (2007)

11. Foryś, U., Bodnar, M.: Time delays in proliferation process for solid avascular tumour. Math. Comput. Model. 37, $1201-1209(2003)$

12. Friedman, A., Lam, K.: Analysis of a free-boundary tumor model with angiogenesis. J. Differ. Equ. 259, 7636-7661 (2015)

13. Friedman, A., Reitich, F.: Analysis of a mathematical model for the growth of tumors. J. Math. Biol. 38, 262-284 (1999)

14. Fu, S., Cui, S.: Global existence and stability of solution of a reaction-diffusion model for cancer invasion. Nonlinear Anal., Real World Appl. 10, 1362-1369 (2009)

15. Greenspan, H.: Models for the growth of solid tumor by diffusion. Stud. Appl. Math. 51, 317-340 (1972)

16. Greenspan, H.: On the growth and stability of cell cultures and solid tumors. J. Theor. Biol. 56, 229-242 (1976)

17. Piotrowska, M.J.: Hopf bifurcation in a solid avascular tumor growth model with two discrete delays. Math. Comput. Model. 47, 597-603 (2008)

18. Ragusa, M.A., Russo, G.: ODEs approaches in modeling fibrosis. Comment on "Towards a unified approach in the modeling of fibrosis: a review with research perspectives" by Martine Ben Amar and Carlo Bianca. Phys. Life Rev. 17, $112-113(2016)$

19. Shen, H., Wei, X.: A qualitative analysis of a free boundary problem modeling tumor growth with angiogenesis. Nonlinear Anal., Real World Appl. 47, 106-126 (2019)

20. $\mathrm{Wu}, \mathrm{J}$., Zhou, F.: Asymptotic behavior of solutions of a free boundary problem modeling tumor spheroid with Gibbs-Thomson relation. J. Differ. Equ. 262, 4907-4930 (2017)

21. $\mathrm{Xu}, \mathrm{S}$., Feng, Z: Analysis of a mathematical model for tumor growth under indirect effect of inhibitors with time delay in proliferation. J. Math. Anal. Appl. 374, 178-186 (2011)

22. Yi, T., Huang, L.: Generalization of the Bernfeld-Chaddock conjecture and its proof. Acta Math. Sinica (Chin. Ser.) 50, $261-270$ (2007)

23. Zhuang, Y., Cui, S.: Analysis of a free boundary problem modeling the growth of multicell spheroids with angiogenesis. J. Differ. Equ. 265, 620-644 (2018)

\section{Submit your manuscript to a SpringerOpen ${ }^{\circ}$ journal and benefit from:}

- Convenient online submission

- Rigorous peer review

- Open access: articles freely available online

- High visibility within the field

Retaining the copyright to your article

Submit your next manuscript at $\gg$ springeropen.com 